\title{
Generic Single-Channel Detection of Absence Seizures
}

Petersen, Eline B.; Duun-Henriksen, Jonas; Mazzaretto, Andrea; Kjær, Troels W.; Thomsen, Carsten E.; Sørensen, Helge Bjarup Dissing

\section{Published in:}

Proceedings of the 33rd Annual International Conference of the IEEE EMBS

Link to article, DOI:

10.1109/IEMBS.2011.6091194

Publication date:

2011

Link back to DTU Orbit

Citation (APA):

Petersen, E. B., Duun-Henriksen, J., Mazzaretto, A., Kjær, T. W., Thomsen, C. E., \& Sørensen, H. B. D. (2011). Generic Single-Channel Detection of Absence Seizures. In Proceedings of the 33rd Annual International Conference of the IEEE EMBS (pp. 4820-4823). IEEE. https://doi.org/10.1109/IEMBS.2011.6091194

\section{General rights}

Copyright and moral rights for the publications made accessible in the public portal are retained by the authors and/or other copyright owners and it is a condition of accessing publications that users recognise and abide by the legal requirements associated with these rights.

- Users may download and print one copy of any publication from the public portal for the purpose of private study or research.

- You may not further distribute the material or use it for any profit-making activity or commercial gain

- You may freely distribute the URL identifying the publication in the public portal 


\title{
Generic Single-Channel Detection of Absence Seizures
}

\author{
Eline B. Petersen ${ }^{a}$, Jonas Duun-Henriksen ${ }^{a, c}$, Andrea Mazzaretto ${ }^{a}$, Troels W. Kjær ${ }^{b}$, Carsten E. Thomsen $^{d}$ \\ and Helge B. D. Sorensen ${ }^{a}$
}

\begin{abstract}
A long-term EEG-monitoring system, which automatically marks seizure events, is useful for diagnosing and treating epilepsy. A generic method utilizing the low interand intra-patient variabilities in EEG-characteristics during absence seizures is proposed. This paper investigates if the spike-and-wave behaviour during absence seizures is so distinct that a single-channel implementation is possible. 18 channels of scalp electroencephalography (EEG), from 19 patients suffering from childhood absence epilepsy, are analysed individually. The characteristics of the seizures are captured using the energy content of wavelet transform subbands and classified using a support vector machine. To ease the evaluation of the method, we present a new graphical visualization of the performance based on the topographical distribution on the scalp.

The presented seizure detection method shows that the best result is obtained for the derivation F7-FP1. Using this channel a sensitivity of $99.1 \%$, positive predictive value of $94.8 \%$, mean detection latency of $3.7 \mathrm{~s}$, and false detection rate value of $0.5 / \mathrm{h}$ was obtained. The topographical visualization of the results clearly shows that the frontal, midline, and parietal channels outperform detection based on the channels in the occipital region.
\end{abstract}

\section{INTRODUCTION}

The incidence of childhood absence epilepsy (CAE) is reported as high as 9.6/100.000 in children younger than 15 years of age [1]. CAE has its onset in children age 4-10 years and causes the patient to experience frequent seizures up to hundreds a day. The clinical manifestation of an absence seizure is loss of conciousness and for some this is accompanied by eye blinking or lip smacking, but often no physical signs are observed. Seizures without clinical manifestations are termed paroxysms, but for convenience we use the term seizures for all epileptic activity. Long-term monitoring of CAE patients is highly relevant since the large number of seizures and sparse physical manifestation make it difficult to otherwise recognize absences for diagnostic and treatment purposes.

Absence seizures are very characteristic in the discharge of $3 \mathrm{~Hz}$ spike-and-wave complexes [2]. The duration of a seizure is reported to vary from a few seconds to half a minute. Furthermore, the patients also experience bursts of spike-and-wave complexes of shorter duration which do not affect the consciousness. Generally, seizures of a duration

Corresponding authors: Eline B. Petersen, Elinebp@hotmail.com, and Helge B. D. Sorensen, hbselektro.dtu.dk

${ }^{a}$ Department of Electrical Engineering, Technical University of Denmark, ØrstedsPlads, building 349, DK-2800 Kgs.Lyngby

${ }^{b}$ Department of Clinical Neurophysiology NF-3063, Rigshospitalet University Hospital, Blegdamsvej 9, DK-2100 Copenhagen

${ }^{c}$ Hypo-Safe A/S, Diplomvej 381, DK-2800 Kgs.Lyngby

${ }^{d}$ Department of Oral Medicine, University of Copenhagen, Nørre Alle 20, DK-2200 Copenhagen shorter than 3 seconds have no impact on the consciousness of the patient [2].

In the field of seizure detection, patient-specific methods are generally reported to have a higher performance compared to generic methods [5] . However, generic methods are easier and faster to apply and therefore more relevant for clinical use. The characteristics of epileptic seizures of the same type are generally described to have large inter-patient variability, but small intra-patient variability [3], which is why patient-specific methods often outperform generic. However, patients suffering from CAE have relatively little inter-patient variability in the seizure characteristics [4].

A generic method of automatic detection of typical absence seizures is prosed for EEG monitoring of CAE patients. Due to the very characteristic nature of the absence seizures it is investigated if a method based on single-channel EEG is feasible. As stated, only seizures longer than 3 seconds have an impairing effect on the patients consciousness. To ensure that all impairing seizures are detected, we aim at detecting all seizures with a duration longer than 2 seconds. The result of the single-channel implementation is presented graphically to ease the topographical evaluation of the performance.

\section{Method}

\section{A. Clinical Data}

In this study scalp-EEG from 19 CAE patients (10 females, 9 males), with a mean age of $7.6 \pm 1.7$ years. 24 recordings with a total duration of 11 hours and 48 minutes, containing a total of 177 seizures, the 111 seizures with a duration longer than 2 seconds are detectable, see Section II-D. Data were recorded using a Cadwell Easy II from Cadwell Laboratories ${ }^{\mathrm{TM}}$ as part of routine examinations performed at the Department of Clinical Neurophysiology at the Rigshospitalet University Hospital. The electrodes are placed according to the international 10-20 system and the 18 EEG channels are extracted with a transversal montage. The recordings are sampled with a frequency of $200 \mathrm{~Hz}$, bandpass filtered from $0.53-70 \mathrm{~Hz}$, and notch filtered to remove $50 \mathrm{~Hz}$ power-line noise.

\section{B. Data Processing}

Each EEG-channel is analysed in extracted epochs of 2 seconds (400 samples) overlapping with 1 second (50\%). For each epoch, features are calculated, classified, and processed by temporal constraints before the performance is calculated. Figure 1 shows the flow diagram for the seizure detection algorithm. 


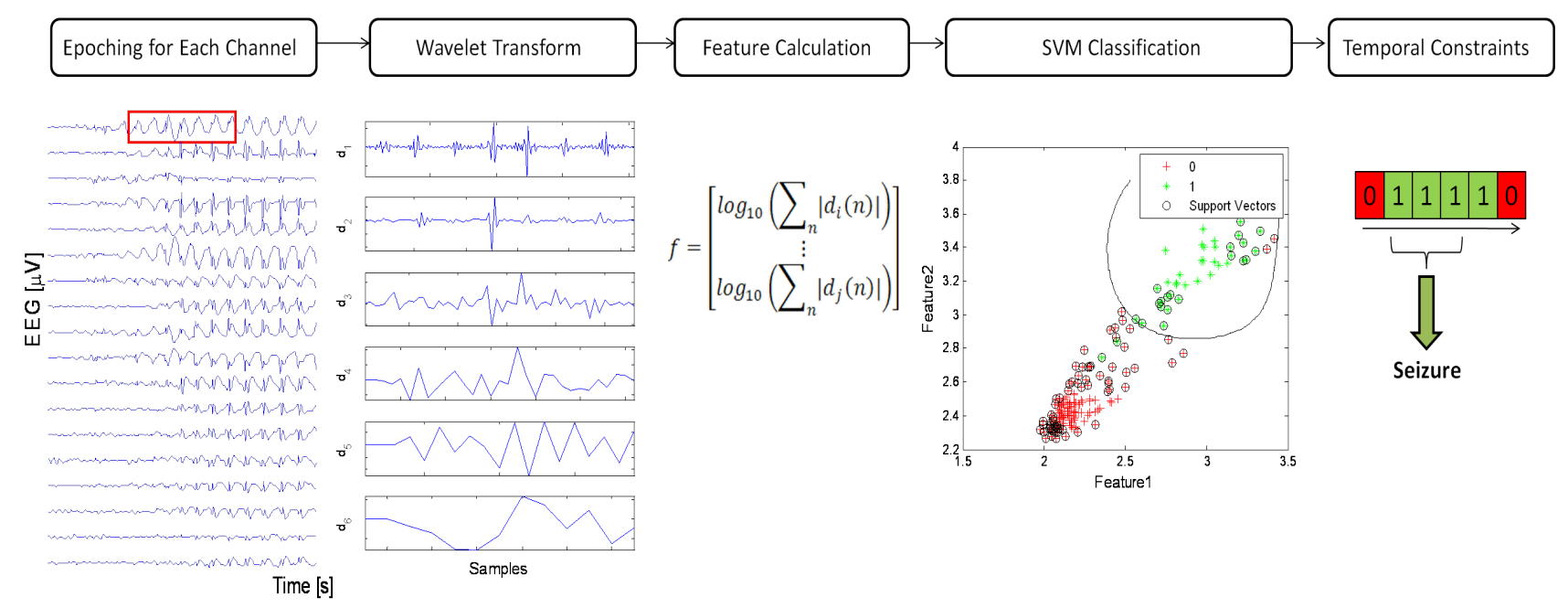

Fig. 1. Flow diagram of the automatic seizure detection scheme. 2-second epochs from each of the 18 EEG-channels are extracted and a 6-level wavelet decomposition made. The log-sum energy is calculated based on the wavelet subbands and classified using a support vector machine with a non-linear radial basis kernel. A temporal constraint is made so that a seizure is not detected before three consecutive epochs is classified as such. The red examples with the value 0 indicates normal epochs and green examples with value 1 are seizure epochs.

1) Feature Calculation: For seizure detection a feature is a distinct property or characteristic which clearly separates normal and ictal EEG. Studies such as [5] and [6] have proven the strength of using the energy of wavelet transform subbands for patient-specific seizure detection. This study will be based on the same approach, but changed slightly to favour generic detection of absences.

The frequency analysis was performed based on the wavelet transform (WT) rather than the traditionally used short time Fourier transform (STFT). In the STFT the constant-length window, used for the analysis, leads to a constant frequency resolution, which is not optimal for all frequencies. The WT, however, varies the window length to provide poor frequency resolution for high frequencies, but good resolution for low frequencies. Since time and frequency resolution are inversely proportional, a good frequency resolution is provided by a poor time resolution and vice versa. Another difference is found in the choice of basisfunction; where STFT decomposes the signal into sinusoid functions, the WT uses a mother wavelet which is scaled and shifted in time in order to decompose the signal into subbands of different frequency content. The discrete WT is implemented using high- and low-pass filters which are characterized by the chosen mother wavelet. These filters decompose the signal into low frequency approximation bands, $a_{L}$, and high frequency detail bands, $d_{L}$. For each level, $L$, of the WT the approximation band can be further decomposed into a detail and an approximation band, $d_{L+1}$ and $a_{L+1}$, see Figure 2. At each level, the frequency content is bisected, and therefore the number of data points can also be down-sampled with a factor two without loosing information.

2) Log-Sum Energy: Because the coefficients of the detail bands are highly sensitive to noise, these are rarely used as

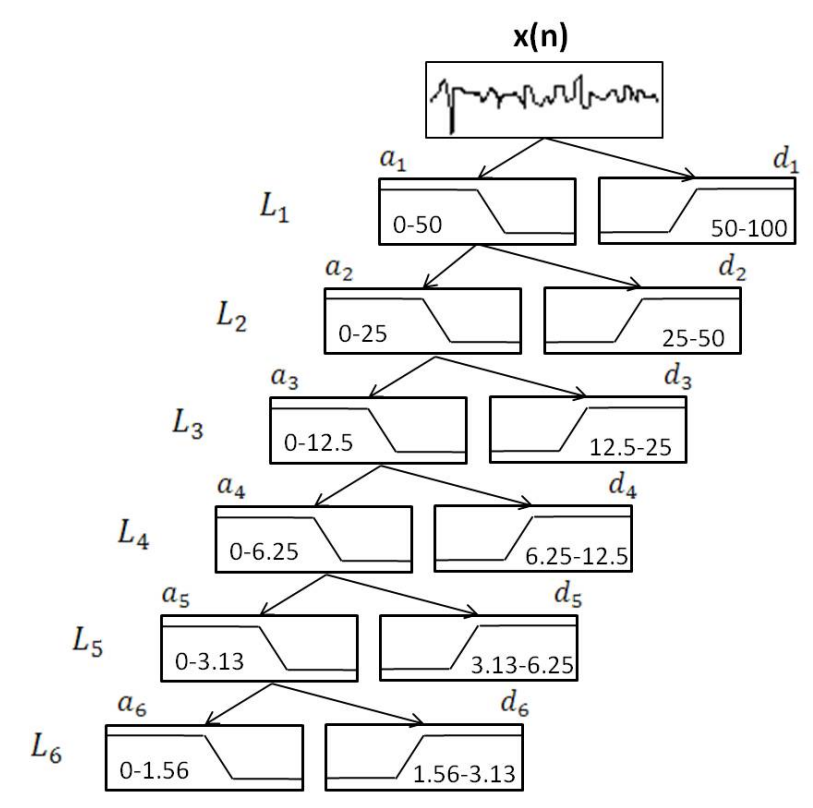

Fig. 2. Wavelet decomposition of the EEG signal $x(n)$, sampled at 200 $\mathrm{Hz}$, into 6 levels. At each level $L$ an approximation band, $a_{L}$, and a detail band $d_{L}$ are generated by low- and high-pass filtering, respectively. The numbers above indicate the frequency range contained in the approximation and detail bands.

features. Shoeb et. al. [5] suggested applying the log-sum energy of the subband coefficients as features:

$$
f(\text { epoch })=\left[\begin{array}{c}
\log _{10}\left(\sum_{n}\left|d_{i}(n)\right|\right) \\
\vdots \\
\log _{10}\left(\sum_{n}\left|d_{j}(n)\right|\right)
\end{array}\right]
$$

where $n$ runs over the WT coefficients, $i$ is the lowest subband included, and $j$ is the highest subband included. A 


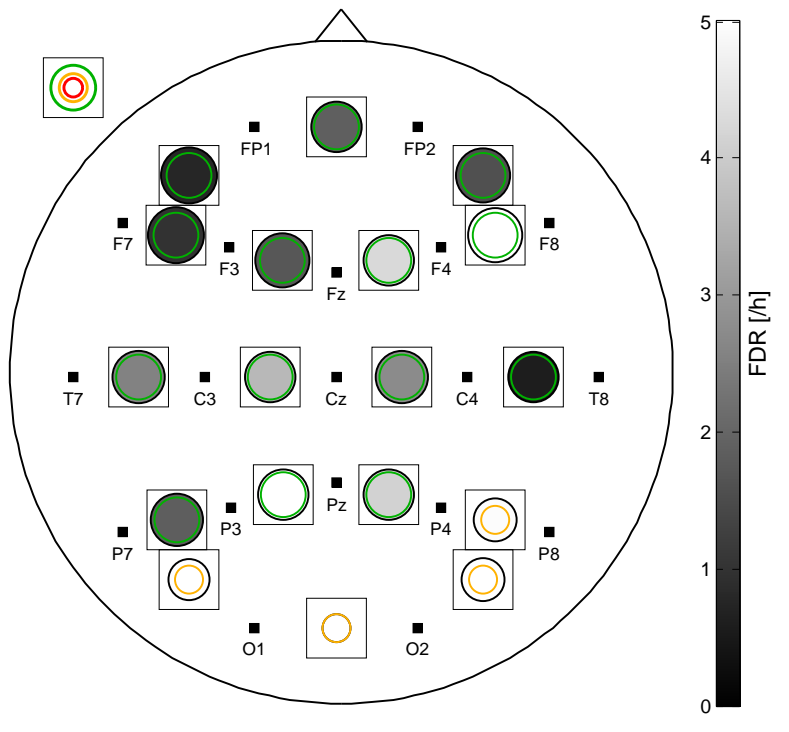

Fig. 3. Topographical distribution of the overall performance. Each EEGelectrode is marked with a black square. The performance of a given channel is located between the two electrodes from which they are made. For each channel both the sensitivity and FDR are specified. The sensitivity is illustrated by the diameter of the circle, with the rectangle indicating $100 \%$. The sensitivities are scaled exponentially. The colored circles marks the sensitivities of $50 \%$ (red), $70 \%$ (yellow), and $90 \%$ (green). The FDR is interpreted from the grey level of the circle, the value of the FDR is shown in the colorbar on the right.

non-linear scaling of the energy using the logarithm amplifies small energy differences to better separate seizure and nonseizure activity.

Shoeb et. al. applied a 7-level WT using a Daubechies 4 (Db4) mother wavelet at a sampling frequency of $256 \mathrm{~Hz}$ and calculated the energy of subbands $d_{4}-d_{7}$ containing frequencies from 1 to $16 \mathrm{~Hz}$. Since this study works with a different kind of epilepsy and with a lower sampling frequency, the optimal choice of subbands may also differ. Different combinations of subbands were investigated and the results showed that including more subbands improved the performance. The best performance was found using the subbands $d_{1}-d_{6}$.

\section{Feature Classification}

The binary classification problem of determining whether an epoch belongs to the class of normal or ictal is solved by using a support vector machine (SVM). The SVM is a supervised learning approach that conveys the classification problem into a convex optimization problem such that a global optimum exists [7]. The classification works in two steps; a learning and a test step. In the learning step a decision boundary is created based on training data. This decision boundary is then applied in the test step to classify each epoch of the test data.

The linear SVM will create a linear decision boundary by maximizing the distance between the boundary cases (the support vectors) of each class. When non-linear trends are present in the data, as in the case of this study, a non-linear
TABLE I

PERFORMANCE OF THE SEIZURE DETECTION FOR EACH OF THE 18 CHANNELS. THE LINES INDICATE THE SEPARATION BETWEEN FRONTAL, MIDLINE, PARIETAL, AND OCCIPITAL CHANNELS. THE BEST RESULTS ARE UNDERLINED.

\begin{tabular}{l||cccc} 
Channel & SE $(\%)$ & FDR $(/ \mathrm{h})$ & PPV $(\%)$ & Latency $(\%)$ \\
\hline \hline F7-FP1 & $\underline{99.1}$ & $\underline{0.5}$ & $\underline{94.8}$ & $\frac{3.7}{3.9}$ \\
FP1-FP2 & 93.7 & 1.4 & 86.7 & 3.7 \\
FP2-F8 & 96.4 & 0.6 & 93.9 & 3.9 \\
F7-F3 & 99.1 & 1.0 & 90.2 & 3.8 \\
F3-FZ & 97.3 & 1.4 & 86.4 & 3.7 \\
FZ-F4 & 92.8 & 2.1 & 80.5 & 3.7 \\
F4-F8 & 97.3 & 2.7 & 77.1 & 3.9 \\
\hline T7-C3 & 95.5 & 2.1 & 80.9 & 3.6 \\
C3-CZ & 91.9 & 2.3 & 79.1 & 4.0 \\
CZ-C4 & 92.8 & 2.1 & 80.5 & 3.8 \\
C4-T8 & 93.7 & 0.6 & 93.7 & 3.7 \\
\hline P7-P3 & 96.4 & 1.5 & 85.6 & 3.7 \\
P3-PZ & 89.2 & 2.6 & 76.2 & 4.0 \\
PZ-P4 & 91.0 & 2.7 & 75.9 & 4.0 \\
P4-P8 & 91.0 & 1.8 & 82.8 & 4.0 \\
\hline P7-O1 & 85.6 & 4.1 & 66.0 & 4.3 \\
O1-O2 & 68.5 & 6.9 & 48.4 & 4.1 \\
O2-P8 & 86.5 & 3.9 & 67.6 &
\end{tabular}

version of the SVM is applied. The non-linear SVM uses a kernel function to project the data into a higher-dimensional space where a linear decision boundary can successfully separate the two classes. The SVM ${ }^{\text {light }}$ package [8] is used with a non-linear radial basis kernel with the width $\gamma$ set to 1.1. The SVM parameters $J$ and $C$ define the cost by which the training errors on positive examples outweigh errors on negative examples and the trade-off between training errors and margin, respectively. The parameters are set to $J=1.3$ and $C=1$. Originally, the SVM-parameters were to be optimized for each EEG-channel individually, but extensive analysis revealed that the optimal parameter choice does not change significantly between channels.

The test and training data are separated using the leaveone-out approach such that data from one patient is tested using an SVM trained on all of the remaining data. Due to the higher amount of normal data a ratio $r$ between the number of non-ictal and ictal epochs is chosen to reduce the size of the training set and thereby the computation time. The value of $r$ is optimized to ensure that enough normal epochs are included to generate a satisfactory decision boundary. The optimum is found for $r=10$.

\section{Temporal Constraints}

To avoid a high number of false detections the result from the SVM classification is adjusted by temporal constraints. We wish to avoid detections due to seizure-like activity of short duration, but still detect all seizures with a duration longer than 2 seconds. This is obtained by incorporating a temporal constraint so that at least 3 consecutive ( 2 seconds) epochs must be classified as containing seizure activity before a seizure is detected. However, when examining the result it was seen that some seizures shorter than 2 seconds were recognized by the algorithm as 3 consecutive seizure epochs. These short seizures do not affect the patient and are 
therefore not the main interest for the neurophysiologist and can be disregarded. However, the method is not wrong in recognizing the epochs as containing seizure activity. This issue was dealt with by allowing the algorithm to detect seizures, but not including them as true positive events if they are detected or as false positives if they are not.

Four performance measures are calculated; sensitivity (SE), the number of false detections per hours (FDR), mean detection latency, and positive predictive value (PPV) denoting the proportion of true seizures in the total number of detections.

\section{RESUlTS AND DISCUSSION}

The four performance measures for each of the 18 channels are shown in Table I. The corresponding topographical visualization of the performance is shown in Figure 3, where the color of the circles indicates the FDR, and the radius shows the sensitivity. The radius is scaled exponentially to emphasise differences between high sensitivities.

The best performance is found for the electrodes in the frontal region. The best overall performance is found in the channel F7-FP1. The sensitivity of F7-FP1 is matched in the other channel incorporating F7, while the FDR, PPV, and latency are higher. Generally channels located in the frontal, midline, and parietal regions show a better performance when compared to the channels in the occipital region. This is in accordance with the biological findings that spike-and-wave complexes during an absence peak in the frontal regions, but also have a high activity over the midline region [9].

The FDR for all the channels are high, but it should be noted that the EEG recordings are of short duration (mean duration 29 minutes and 32 seconds). This means that a single false detection will result in an FDR of $2 / \mathrm{h}$. It should also be kept in mind that the CAE patients experience a high number of seizures. One patient has 13 seizures (23 including seizures shorter than 2 seconds) in a 30 minute recording. The PPV takes this into account by describing the percentage of all detection that is in fact true seizures. A PPV of $94.8 \%$ is found in F7-FP1 meaning that only $5.2 \%$ of all detected seizures are false detections.

Generally very few studies focus on generic detection of absence seizures; only one directly comparable study was found [10]. Here the EEG is decomposed into half-waves and classified using thresholding of different half-wave features. The method is based on three channels, but allows detection of seizures from just one channel, the result is given as a mean for different combinations of channels. They obtained a sensitivity of $70 \%$ and an FDR varying from $2.4-10 / \mathrm{h}$. In comparison, our best result is obtained in the channel F7-FP1 with a sensitivity of $99.1 \%$ and an FDR of $0.5 / \mathrm{h}$.

\section{CONCLusion And Future Work}

\section{A. Conclusion}

The very characteristic spike-and-wave discharges during absence seizures were the basis of developing a singlechannel generic method for seizure detection. Based on an approach successfully applied for multi-channel patientspecific seizure detection we obtained a good result using a single-channel generic implementation. High sensitivities and low mean detection delays were obtained. The high PPV values indicate that although the FDR are high, the number of false detections relative to the number of true detections are low. The overall best result is obtained for the frontal channel F7-FP1 with a sensitivity of $99.1 \%$, an FDR of 0.5 , a PPV of $94.8 \%$, and a mean detection delay of $3.7 \mathrm{~s}$. The novel topographical visualization of the performance measure eased the interpretation of the result and clearly showed that channels in the frontal, midline, and parietal regions performed better than channels in the occipital region. Comparing the result to a similar method, [10], we obtained better sensitivity for all the channels and a better or matching FDR for all the channels.

\section{B. Future Work}

One task which should be looked into is to reduce the number of false detections. This could be done including an additional feature relating more to the size of the amplitude than the frequency content.

Two other idiopathic generalized epilepsies exist, the juvenile absence epilepsy (JAE) and the juvenile myoclonic epilepsy (JME), in which absence seizures are seen. These absences are described to be less 'pure' than the CAE absences [2]. However, it could be interesting to apply the method described here to investigate if it is equally successful in detecting these seizures and if the topographical distribution vary between the different epilepsies.

\section{REFERENCES}

[1] P. Loiseau and C. P. Panayiotopoulos, "Childhood absence epilepsy", International League Against Epilepsy (ILAE), 2002.

[2] P.L. Pearl and G.L. Holms, "Absence Seizures", in Pediatric Epilepsy: Diagnosis and Therapy, Demos, 2008, chapter 22.

[3] I. Soltesz and K. Staley, "Computational Neuroscience in Epilepsy", Elsevier Inc, 2008, chapters 28 and 32.

[4] G. Baier, T. Hermann, S. Sahle and U. Stephani, "Sonified Epileptic Rythms", in Preceedings of the 12th International Conference on Auditory Display, London, UK, 2006, pp. 148-151.

[5] A. Shoeb, H. Edwards, J. Connolly, B. Bourgeois, S.T. Treves and J. Guttag, "Patient-Specific Seizure Onset Detection", Epilepsy \& Behavior, vol. 5, 2004, pp 483-493.

[6] J. Henriksen, L.S. Remvig, R.E. Madsen, I. Conradsen, T.W. Kjær and H.B.D. Sorensen. "Automatic Seizures Detection: Going from sEEG to iEEG", in 32nd Annual Internatinoal Conference of the IEEE EMBS, 2010, pp 2431-2434.

[7] C. M. Bishop. "Pattern Recognition and Machine Learning", Springer, 2006.

[8] T. Joachims, "Learning to classify text using support vector machines: Methods, theory, and algorithms", Computational Lingustics, vol. 29, 2002, pp 655-661.

[9] R. Ferri, G. Iliceto, and V. Carlucci. "Topographic EEG Mapping of 3/s Spike-and-Wave Complexes during Absence Seizures".The Italian Journal of Neurological Sciences, vol. 16, 1995, pp 541-547.

[10] D.J. Koffler and J. Gotman "Automatic Detection of Spike-and-Wave Bursts in Ambulatory EEG Recordings". Electroencephalography and clinical Neurophysiology, vol. 61, 1985, pp 165-180. 